\title{
The Erdős-Ko-Rado Theorem for 2-pointwise and 2-setwise intersecting permutations
}

\author{
Karen Meagher * A. Sarobidy Razafimahatratra \\ Department of Mathematics and Statistics \\ University of Regina \\ Regina, Saskatchewan, Canada \\ \{karen.meagher, sarobidy\}@uregina.ca
}

Submitted: Apr 30, 2020; Accepted: Sep 27, 2021; Published: Oct 22, 2021

(C) The authors. Released under the CC BY-ND license (International 4.0).

\begin{abstract}
In this paper we consider the conjectured Erdős-Ko-Rado property for 2-pointwise and 2-setwise intersecting permutations.

Two permutations $\sigma, \tau \in \operatorname{Sym}(n)$ are $t$-setwise intersecting if there exists a $t$ subset $S$ of $\{1,2, \ldots, n\}$ such that $S^{\sigma}=S^{\tau}$. Further, two permutations $\sigma, \tau \in$ $\operatorname{Sym}(n)$ are $t$-pointwise intersecting if there exists a $t$-subset $S$ of $\{1,2, \ldots, n\}$ such that $s^{\sigma}=s^{\tau}$ for each $s \in S$. A family of permutations $\mathcal{F} \subset \operatorname{Sym}(n)$ is called $t$ setwise (resp. $t$-pointwise) intersecting, if any two permutations in $\mathcal{F}$ are $t$-setwise (resp. $t$-pointwise) intersecting. We say that $\operatorname{Sym}(n)$ has the $t$-setwise intersecting property if for any family $\mathcal{F}$ of $t$-setwise intersecting permutations, $|\mathcal{F}| \leqslant t !(n-t)$ !. Similarly, $\operatorname{Sym}(n)$ has the $t$-pointwise intersecting property if for any family $\mathcal{F}$ of $t$-pointwise intersecting permutations, $|\mathcal{F}| \leqslant(n-t)$ !.

Ellis (["Setwise intersecting families of permutations". J. Combin. Theory Ser. $A, 119(4): 825-849,2012])$, proved that if $n$ is sufficiently large relative to $t$, then $\operatorname{Sym}(n)$ has the $t$-setwise intersecting property. Ellis also conjectured that this result holds for all $n \geqslant t$. Ellis, Friedgut and Pilpel ["Intersecting families of permutations." J. Amer. Math. Soc. 24(3):649-682, 2011] also proved that for $n$ sufficiently large relative to $t, \operatorname{Sym}(n)$ has the $t$-pointwise intersecting property. It is also conjectured that $\operatorname{Sym}(n)$ has the $t$-pointwise intersecting property for $n \geqslant 2 t+1$. In this work, we prove these two conjectures for $\operatorname{Sym}(n)$ when $t=2$.
\end{abstract} Mathematics Subject Classifications: 05C35, 05C69, 20B05

*Research supported in part by an NSERC Discovery Research Grant, Application No.: RGPIN03852-2018. 


\section{Introduction}

The study of intersecting properties of finite sets is a central theme in extremal combinatorics. A collection or family of subsets $\mathcal{F}$ of $\{1,2, \ldots, n\}$ is called intersecting if for any $A, B \in \mathcal{F}, A \cap B \neq \varnothing$. In 1961, Erdös, Ko and Rado proved an important result on intersecting families of $k$-subsets of $\{1,2, \ldots, n\}$. This result is stated in the following theorem. We denote the collection of all $k$-subsets of $\{1,2, \ldots, n\}$ by $\left(\begin{array}{c}{[n]} \\ k\end{array}\right)$.

Theorem 1 (Erdös, Ko, Rado [9]). For any positive integer $n$ and $k$ such that $n \geqslant 2 k$, if $\mathcal{F}$ is an intersecting family of $\left(\begin{array}{c}{[n]} \\ k\end{array}\right)$, then $|\mathcal{F}| \leqslant\left(\begin{array}{c}n-1 \\ k-1\end{array}\right)$. Moreover, if $n>2 k$, then

$|\mathcal{F}|=\left(\begin{array}{c}n-1 \\ k-1\end{array}\right)$ if and only if $\mathcal{F}=\mathcal{F}_{i}=\left\{A \in\left(\begin{array}{c}{[n]} \\ k\end{array}\right) \mid i \in A\right\}$, for some $i \in\{1,2, \ldots, n\}$.

There are several proofs and extensions of Theorem 1 in the literature $[4,5,7-9,11,12$, 16]. In particular, Deza and Frankl [11] extended Theorem 1 for permutations. A family of permutations $\mathcal{F} \subseteq \operatorname{Sym}(n)$ is called $t$-pointwise intersecting if for any $\sigma, \tau \in \mathcal{F}$, there exists distinct $i_{1}, i_{2}, \ldots, i_{t} \in\{1,2, \ldots, n\}$ such that $i_{\ell}^{\sigma}=i_{\ell}^{\tau}$, for all $\ell \in\{1,2, \ldots, t\}$. It is proven in [11] that if $\mathcal{F}$ is a family of 1 -pointwise intersecting permutations of the symmetric group $\operatorname{Sym}(n)$, then $|\mathcal{F}| \leqslant(n-1)$ !. In 2003, Cameron and $\mathrm{Ku}$ [2], independently Larose and Malvenuto [17], proved that the only intersecting families of permutations meeting the bound are cosets of a stabilizer of a point of $\operatorname{Sym}(n)$. In 2009, Godsil and Meagher [13] gave an algebraic proof of this result; the work in this paper uses a similar algebraic approach.

We can also consider intersecting families of permutations from a specific permutation group, rather than all of $\operatorname{Sym}(n)$. For an arbitrary $t$, we say that a permutation group $G$ has the $t$-pointwise intersecting property if any family $\mathcal{F}$ of $t$-pointwise intersecting permutations, is no larger than the maximum size of a pointwise stabilizer of a $t$-set. Deza and Frankl's result [11] proves that $\operatorname{Sym}(n)$ has the 1-pointwise intersecting property.

A natural extension of this type of result is to consider the setwise action of the permutation group. The family of permutations $\mathcal{F}$ is called $t$-setwise intersecting if for any $\sigma, \tau \in \mathcal{F}$, there exists $S \in\left(\begin{array}{c}{[n]} \\ t\end{array}\right)$ such that $S^{\sigma}=S^{\tau}$. The stabilizer of a $t$-set is an example of a $t$-setwise intersecting family of permutations. We say that a permutation group $G$ has the $t$-setwise intersecting property if any family $\mathcal{F}$ of $t$-setwise intersecting permutations, is no larger than the maximum size of a setwise stabilizer of a $t$-set.

Note that 1-pointwise intersecting and 1-setwise intersecting are equivalent, so we simply call this property intersecting.

In particular, $\operatorname{Sym}(n)$ has the $t$-setwise (resp. $t$-pointwise) intersecting property if for any family $\mathcal{F}$ of $t$-setwise (resp. $t$-pointwise) intersecting permutations, $|\mathcal{F}| \leqslant t$ ! $(n-t)$ ! (resp. $|\mathcal{F}| \leqslant(n-t) !)$.

It was also conjectured in [11] that for $n$ sufficiently large with respect to $t$, a $t$ pointwise intersecting family $\mathcal{F}$ of $\operatorname{Sym}(n)$ is such that $|\mathcal{F}| \leqslant(n-t)$ !. This conjecture was proved by Ellis et al. [8] using spectral methods and the representation theory of the symmetric group.

Theorem 2 (Ellis, Friedgut, Pilpel [8]). For $n$ sufficiently large with respect to $t$, if a family of permutations $\mathcal{F}$ of $\operatorname{Sym}(n)$ is t-pointwise intersecting, then $|\mathcal{F}| \leqslant(n-t)$ !. 
Moreover, $|\mathcal{F}|=(n-t)$ ! if and only if $\mathcal{F}$ is a coset of the pointwise stabilizer of $t$ elements from $\{1,2, \ldots, n\}$.

There is a similar result for $t$-setwise intersection. It was proved in 2011 by Ellis [7].

Theorem 3 (Ellis, [7]). For $n$ sufficiently large with respect to $t$, if a family of permutations $\mathcal{F}$ of $\operatorname{Sym}(n)$ is $t$-setwise intersecting, then $|\mathcal{F}| \leqslant t !(n-t)$ !. Moreover, $|\mathcal{F}|=t !(n-t)$ ! if and only if $\mathcal{F}$ is a coset of a stabilizer of a $t$-subset of $\{1,2, \ldots, n\}$.

The proof of Theorem 3 uses similar arguments to the proof of the Deza-Frankl conjecture in [8]. In both proofs, the result holds for $n$ sufficiently large relative to $t$ and exact bounds for $n$ are not given. It is conjectured that for $t$-pointwise intersection the correct lower bound on $n$ is $2 t+1$, while for $t$-setwise intersection it is conjectured in [7] that the result holds for all $n \geqslant t$.

In this paper we will prove that the conjectured exact lower bound on $n$ hold in both cases for $t=2$. We also give a characterization of the sets that meet the bound. Before we can describe this characterization, we need to define some terms.

The regular module of $\operatorname{Sym}(n)$ is the complex vector space with basis $\operatorname{Sym}(n)$ - the elements of this module can be thought of as vectors of length $|\operatorname{Sym}(n)|$. For example, the characteristic vector for a subset $S \subset \operatorname{Sym}(n)$ is the length- $|\operatorname{Sym}(n)|$ vector with the $g$-entry equal to 1 if $g \in S$ and 0 otherwise is, a vector in this module. This module can be identified with the vector space $\mathbb{C}[\operatorname{Sym}(n)]$ which has the structure of a left $\mathbb{C}[\operatorname{Sym}(n)]$ module by left multiplication. So $\mathbb{C}[\operatorname{Sym}(n)]$ can also be identified with a subalgebra of the $|\operatorname{Sym}(n)| \times|\operatorname{Sym}(n)|$-matrices.

It is well-known that each irreducible character of $\operatorname{Sym}(n)$ corresponds to a partition $\lambda \vdash n$ (denoted by $\chi^{\lambda}$ ) For each such irreducible character, let $E_{\lambda}$ be the $|\operatorname{Sym}(n)| \times$ $|\operatorname{Sym}(n)|$-matrix with the $(g, h)$-entry equal to $\chi^{\lambda}\left(h g^{-1}\right)$. We call the image $E_{\lambda}$ (considered as a linear operator on $\mathbb{C}[\operatorname{Sym}(n)])$ the $\lambda$-module and denote it by $V_{\lambda}$.

In [13] it is shown that the characteristic vector of any maximum intersecting set in $\operatorname{Sym}(n)$ is a vector in $V_{[n]} \oplus V_{[n-1,1]}$. In general, if $G$ is any finite 2-transitive group, then the permutation module is the sum of two irreducible representations; the trivial representation and one denoted by $\phi$. In [18] it is shown for any 2-transitive group $G$ that the characteristic vector of any maximum intersecting set of permutations in $G$ lies in the sum of the trivial and the $\phi$-module. This is called the EKR-module property.

In this paper, we will determine the modules in which the characteristic vectors of the maximum 2-setwise and 2-pointwise intersecting sets in $\operatorname{Sym}(n)$ lie. We will use spectral methods and the representation theory of the symmetric group in our proof for Theorem 4 and Theorem 5.

We state our theorems on the 2-setwise and 2-pairwise actions as follows.

Theorem 4. Let $n \geqslant 2$. If $\mathcal{F}$ is a family of 2-setwise intersecting permutations, then $|\mathcal{F}| \leqslant 2(n-2)$ !. Moreover, for $n \geqslant 6$, if $S$ is a maximum 2-setwise intersecting family, then its characteristic vector $\chi_{S}$ is in $V_{[n]} \oplus V_{[n-1,1]} \oplus V_{[n-2,2]}$.

Theorem 5. Let $n \geqslant 2$. If $\mathcal{F}$ is a 2-pointwise intersecting family of permutations, then $|\mathcal{F}| \leqslant(n-2)$ !. In addition, for $n \geqslant 4$ if $S$ is a maximum 2-pointwise intersecting family of $\operatorname{Sym}(n)$, then $\chi_{S}$ in $V_{[n]} \oplus V_{[n-1,1]} \oplus V_{[n-2,2]} \oplus V_{[n-2,1,1]}$. 
We organize this paper as follows. In Section 2 and Section 3, we recall some basics on the symmetric group $\operatorname{Sym}(n)$ and combinatorial objects called weighted adjacency matrices. Section 4 is devoted to the proof of Theorem 4. In Section 5, we give the proof for Theorem 5 .

\section{Background}

\subsection{Derangement graphs}

One of the techniques to prove EKR-type theorems for groups is to use the derangement graph. The set of derangements of a permutation group $G \leqslant \operatorname{Sym}(n)$ is the set of all permutations of $G$ without fixed points. We denote by $\operatorname{Der}(G)$ the set of all derangements of the permutation group $G$. The derangement graph $\Gamma_{G}$ is the undirected graph with vertex set $G$, where two permutations $g, h \in G$ are adjacent if and only if $h g^{-1} \in \operatorname{Der}(G)$. For the case where $G$ is symmetric group we denote $\Gamma_{\text {sym }(n)}$ by $\Gamma_{n}$.

If $\mathcal{F}$ is an intersecting family of $G$, then in $\Gamma_{G}$, the elements of $\mathcal{F}$ form an independent set or coclique. We say that a transitive group has the EKR property if the size of a maximum coclique of $\Gamma_{G}$ is equal to $\frac{|G|}{n}$. This is equivalent to the group having the 1-pointwise (or, equivalently, 1-setwise) intersecting property.

Given a graph $X$, we denote by $\alpha(X)$ and $\omega(X)$ respectively the maximum size of a coclique and maximum size of a clique of $X$. The following result is well-known and a proof can be found in [15, Section 2.1].

Lemma 6 (Clique-coclique bound). Let $X$ be a vertex-transitive graph on $n$ vertices. Then

$$
\alpha(X) \omega(X) \leqslant n
$$

Corollary 7. The symmetric group $\operatorname{Sym}(n)$ has the EKR property.

Proof. First we observe that $\omega\left(\Gamma_{n}\right)$ is at most the dimension, which is $n$ in this case. Moreover, the rows of a Latin square of order $n$ correspond to a set of permutations that forms a clique of size $n$ in $\Gamma_{n}$. Using Lemma 6 , we have

$$
\alpha\left(\Gamma_{n}\right) \leqslant \frac{n !}{n}=(n-1) !
$$

Hence, $\operatorname{Sym}(n)$ has the EKR property.

This approach does not work in general for 2-setwise or 2-pointwise intersection. To see this, consider $\operatorname{Sym}(n)$ with 2-pointwise intersection; a clique in the corresponding derangement graph would be a sharply 2-transitive subset of $\operatorname{Sym}(n)$. Since such a set does not exist for all $n$, the clique-coclique bound will not always hold with equality. Rather, we will use a generalization of the ratio bound, as was used in $[7,8,14]$. The ratio 
bound is also known as the Delsarte bound or Hoffman bound, we refer the reader to [15] for a proof. The generalization that we will actually use is a special case of Delsarte's linear programming method originally proved in [3].

For a graph $X$ on $n$ vertices, an $n \times n$ real symmetric matrix $A=\left(a_{i, j}\right)$ with constant row sum is a weighted adjacency matrix (or a pseudo-adjacency matrix) for $X$ if $a_{i, j}=0$ whenever $i \nsim_{X} j$ (note that $a_{i, j}$ could be 0 for adjacent vertices $i$ and $j$ ). We use 1 to denote the all ones vector and $J$ for the all ones matrix (the dimensions will be clear from context).

Theorem 8 (Weighted Ratio Bound). Let $X$ be a connected graph and let $A$ be a weighted adjacency matrix for $X$ with constant row and column sum $d$. If the least eigenvalue of $A$ is $\tau$, then

$$
\alpha(X) \leqslant \frac{|V(X)|}{1-\frac{d}{\tau}} .
$$

Further, if equality holds for some coclique $S$ with characteristic vector $v_{S}$, then

$$
v_{S}-\frac{|S|}{|V(X)|} \mathbf{1}
$$

is an eigenvector with eigenvalue $\tau$.

Proof. Set $v=|V(X)|, s=|S|$, and denote $A(X)$ by $A$. Let $\left\{\mathbf{1}, w_{2}, \ldots, w_{n}\right\}$ be an orthonormal basis of real eigenvectors for $A$ with $A w_{i}=\lambda_{i} w_{i}$.

Define

$$
M=A-\tau I-\frac{d-\tau}{v} J
$$

Then $M \mathbf{1}=0$, and for $w_{i}$

$$
M w_{i}=\left(\lambda_{i}-\tau\right) w_{i}
$$

since $w_{i}$ is orthogonal to the all ones vector. Thus, all the eigenvalues of $M$ are nonnegative, and $M$ is positive semidefinite. Hence, for any vector $x$

$$
0 \leqslant x^{T} M x=x^{T} A x-\tau x^{T} x-\frac{d-\tau}{v} x^{T} J x .
$$

Let $x$ be the characteristic vector of a coclique of size $s$. Then $x^{T} A x=0$ and ( 1$)$ simplifies to

$$
0 \leqslant-\tau s-\frac{d-\tau}{v} s^{2}
$$

Hence

$$
\tau s \leqslant-\frac{d-\tau}{v} s^{2}
$$

and the inequality follows. If equality holds, then $x^{T} M x=0$; since $M$ is positive semidefinite, this implies that $M x=0$. Therefore

$$
(A-\tau I) x=\frac{d-\tau}{v} J x=\frac{s}{v}(d-\tau) \mathbf{1}=(A-\tau I) \frac{s}{v} \mathbf{1} .
$$

Setting $x=v_{S}-\frac{s}{v} \mathbf{1}$ implies the second claim. 


\subsection{Eigenvalues of normal Cayley graphs}

Let $G$ be a group and let $C$ be an inverse-closed subset of $G \backslash\{1\}$. A Cayley graph Cay $(G, C)$ is graph whose vertex set is $G$, and two group elements $g$ and $h$ are adjacent if $h g^{-1} \in C$. A Cayley graph is called normal if the set $C$ is closed under conjugation. That is, for any $g \in G$, we have $g C g^{-1}=C$. The derangement graph for a group is a Cayley graph, in particular, $\Gamma_{G}=\operatorname{Cay}(G, \operatorname{Der}(G))$. Since $\operatorname{Der}(G)$ is closed under conjugation, the derangement graph is a normal Cayley graph. In this subsection, we review some properties of Cayley graphs and give a formula for the eigenvalues of normal Cayley graphs.

The eigenvalues of a normal Cayley graph $\operatorname{Cay}(G, C)$ can be obtained from the irreducible characters of the group $G$. We present this result in the following lemma which is usually attributed to Babai [1], or Diaconis and Shahshahani [6]; a proof may be found in [15, Section 11.12].

Lemma 9. Let $\operatorname{Irr}(G)$ be the set of all irreducible characters on a group $G$. If $X=$ $\operatorname{Cay}(G, C)$ is a normal Cayley graph, then eigenvalues of the adjacency matrix of $X$ are given by

$$
\xi_{\chi}=\frac{1}{\chi(\mathrm{id})} \sum_{g \in C} \chi(g),
$$

where $\chi \in \operatorname{Irr}(G)$. The multiplicity of $\xi$ is $\sum m_{\chi}^{2}$ where $m_{\chi}$ is the dimension of $\chi$, and the sum is taken over all irreducible representations $\chi$ with $\xi_{\chi}=\xi$.

We will use a weighted adjacency matrix that is formed by taking a linear combination of matrices from the conjugacy class association scheme on $\operatorname{Sym}(n)$. Then, we show how to calculate the eigenvalues of such a matrix.

For $\rho$ a partition of $n$, let $C_{\rho}$ represent the conjugacy classes of $\operatorname{Sym}(n)$ with shape $\rho$, and define the $n ! \times n !$ matrix

$$
A_{\rho}[g, h]= \begin{cases}1 & \text { if } h g^{-1} \in C_{\rho}, \\ 0 & \text { otherwise. }\end{cases}
$$

The set of matrices $\mathcal{A}=\left\{A_{\rho}\right\}_{\rho \vdash n}$ form an association scheme called the conjugacy class scheme on $\operatorname{Sym}(n)$ - in particular $A_{\rho}$ is the matrix in this association scheme corresponding to the conjugacy class of permutations with shape $\rho$ (see [15, Section 3.3] for details about this association scheme). Note that the conjugacy class scheme for $\operatorname{Sym}(n)$ is a symmetric association scheme since the conjugacy classes of $\operatorname{Sym}(n)$ are all inverse-closed. For a conjugacy class $C_{\rho}$, the matrix $A_{\rho}$ is the adjacency matrix of $\operatorname{Cay}\left(\operatorname{Sym}(n), C_{\rho}\right)$, and by Lemma 9 the eigenvalues of $A_{\rho}$ are given by $\frac{\left|C_{\rho}\right|}{\chi(\mathrm{id})} \chi\left(g_{\rho}\right)$ where $g_{\rho} \in C_{\rho}$ and $\chi$ is an irreducible representation of $\operatorname{Sym}(n)$.

We will consider weighted adjacency matrices of the derangement graphs of $\operatorname{Sym}(n)$ with the 2-setwise and the 2-pointwise actions. The adjacency matrices we consider have a constant weight on each conjugacy class; hence we only consider matrices in the conjugacy class association scheme for $\operatorname{Sym}(n)$. The next result gives a formula for the eigenvalues of such a weighted adjacency matrix. 
Lemma 10. Let $\left\{C_{\rho} \mid \rho \vdash n\right\}$ be the conjugacy classes of $\operatorname{Sym}(n)$, let $A_{\rho}$ be the matrix of the conjugacy classes scheme of $\operatorname{Sym}(n)$ that corresponds to $C_{\rho}$. Let $A=\sum_{\rho \vdash n} \omega_{\rho} A_{\rho}$, where $\left(\omega_{\rho}\right)_{\rho \vdash n} \subseteq \mathbb{R}$. Then the eigenvalues of $A$ are

$$
\xi_{\chi}=\sum_{\rho \vdash n} \omega_{\rho} \frac{\left|C_{\rho}\right|}{\chi(\mathrm{id})} \chi\left(g_{\rho}\right),
$$

where $g_{\rho} \in C_{\rho}$ and $\chi$ is an irreducible representation of $\operatorname{Sym}(n)$.

If the conjugacy class $C_{\rho}$ is a conjugacy class of derangements under the action of $\operatorname{Sym}(n)$, then it is called a derangement class. For the setwise action on 2-sets, a derangement class is any class $C_{\rho}$ in which $\rho$ has no parts summing to 2 . For the pointwise action, a derangement class is any class with a corresponding partition that contains at most one part of size 1.

\section{Representations of $\operatorname{Sym}(n)$}

In this section we state the results on the representation theory of the symmetric group that we need. We do not prove these results, but we refer the reader to Sagan [19] or another such book.

The dimension of a representation is given by the value of the character on the identity element of the group. For the symmetric group, the dimension of an irreducible representation can be computed via the well-known Hook Length Formula [19, Section 3.10].

If $\lambda \vdash n$, then a pair $(i, j)$ is a node of the Young diagram of $\lambda$ if the path rightward $j$ cells from the top leftmost cell and then downward $i$ cells ends on a cell of $\lambda$. By $(i, j) \in \lambda$, we mean $(i, j)$ is a node of $\lambda$. For a node $(i, j) \in \lambda$ define

$$
H_{i, j}=\{(u, j) \in \lambda \mid i \leqslant u\} \cup\{(i, v) \in \lambda \mid j \leqslant v\}
$$

and $h_{i, j}=\left|H_{i, j}\right|$.

Theorem 11 (Hook Length Formula [10]). Let $\lambda \vdash n$. The dimension of the character $\chi^{\lambda}$ (this is the character corresponding to $\lambda$ ) is

$$
\chi^{\lambda}(\mathrm{id})=\frac{n !}{\prod_{(i, j) \in \lambda} h_{i, j}}
$$

where the product is taken over all nodes in the Young diagram of $\lambda$.

We will need to consider the low-dimensional representations of $\operatorname{Sym}(n)$ separately from the ones with higher dimension. The next result is a statement giving the irreducible representations with low dimension. We will not give a proof of this since a similar result is proved in [15, Lemma 12.7.3].

Lemma 12. Let $n \geqslant 13$. If $\chi^{\lambda}$ is an irreducible representation of $\operatorname{Sym}(n)$ of dimension less than $2\left(\begin{array}{c}n+1 \\ 2\end{array}\right)$, then $\lambda$ is one of the following: $[n],\left[1^{n}\right],[n-1,1],\left[2,1^{n-2}\right],[n-2,2]$, $\left[2^{2}, 1^{n-4}\right],\left[n-2,1^{2}\right]$ or $\left[3,1^{n-3}\right]$. 
Remark 13. Note that the irreducible characters given in Lemma 12 have dimension less than $\left(\begin{array}{l}n \\ 2\end{array}\right)$ (see [15, Lemma 12.7.3]). In fact, it is not hard to verify that they are the only ones of dimension less than $\left(\begin{array}{c}n \\ 2\end{array}\right)$, for $n \geqslant 11$.

Next we state the well-known recursive formula for calculating the value of a character of $\operatorname{Sym}(n)$ on a conjugacy class. Let $\chi_{\rho}^{\lambda}$ be the value of the character $\chi^{\lambda}$ on an element of the conjugacy class $C_{\rho}$. A composition is an unordered partition. We will use brackets for the partitions corresponding to irreducible representations, and parentheses for partitions corresponding to the conjugacy classes of $\operatorname{Sym}(n)$.

Lemma 14 (Murnaghan-Nakayama Rule [19]). If $\lambda \vdash n$ and $\rho$ is a composition of $n$, with $\rho=\left(\rho_{1}, \rho_{2}, \ldots, \rho_{k}\right)$, then

$$
\chi_{\rho}^{\lambda}=\sum_{\xi \in \mathrm{RH}_{\rho_{1}}(\lambda)}(-1)^{\ell \ell(\xi)} \chi_{\rho \backslash \rho_{1}}^{\lambda \backslash \xi},
$$

where $\mathrm{RH}_{\rho_{1}}(\lambda)$ is the set of all rim hooks with $\rho_{1}$ cells of $\lambda$, and $\ell \ell(\xi)$ is the number of rows the rim hook spans minus one.

We will build weighted adjacency matrices for the derangement graphs for $\operatorname{Sym}(n)$ with the setwise and the pointwise action. We will then calculate the eigenvalues of these matrices and prove that the ratio bound holds with equality. To calculate the eigenvalues, we will need to determine the value of the irreducible representations on specific conjugacy classes. In our weighting, we weight many of the conjugacy classes to be 0 , so we only need to consider the values of the irreducible representations that have a non-zero weight. The next result gives the values of the irreducible representation on these specific conjugacy classes.

Lemma 15. The irreducible characters of $\operatorname{Sym}(n)$ that do not vanish on the conjugacy classes $C_{(n)}, C_{(n-1,1)}, C_{(n-3,3)}, C_{(n-4,3,1)}, C_{(n-2,2)}$ and $C_{(n-3,2,1)}$ are given in Table 3.

We end this section with considering two representations of the symmetric group. The Young's subgroup $\operatorname{Sym}(n-2) \times \operatorname{Sym}(2)=\operatorname{Sym}([n-2,2])$ is the setwise stabilizer of a 2 -set. Thus, the setwise action of $\operatorname{Sym}(n)$ on sets of size 2 has permutation character

$$
\operatorname{ind}^{\operatorname{Sym}(n)}\left(1_{\operatorname{Sym}([n-2,2])}\right)=\chi^{[n]}+\chi^{[n-1,1]}+\chi^{[n-2,2]} .
$$

Similarly, the Young's subgroup $\operatorname{Sym}(n-2) \times \operatorname{Sym}(1) \times \operatorname{Sym}(1)=\operatorname{Sym}([n-2,1,1])$ is the pointwise stabilizer of a 2 -set. Thus, the pointwise action of $\operatorname{Sym}(n)$ on sets of size 2 has permutation character

$$
\operatorname{ind}^{\operatorname{Sym}(n)}\left(1_{\operatorname{Sym}([n-2,1,1])}\right)=\chi^{[n]}+2 \chi^{[n-1,1]}+\chi^{[n-2,2]}+\chi^{[n-2,1,1]} .
$$

Both of these decompositions follow from calculating the Kostka numbers.

In the weighted adjacency matrix that we construct, the non-trivial characters in the decomposition of each of the permutation character will be exactly the irreducible characters that achieve the minimum eigenvalue. This fact will show that the characteristic vectors of any maximum coclique will lie in a specific $\operatorname{Sym}(n)$-module. 


\section{Proof of Theorem 4}

In this section we will give the weighted adjacency matrix for the derangement graph of $\operatorname{Sym}(n)$ with the 2-setwise action; this matrix has the form

$$
A=\omega_{1} A_{(n)}+\omega_{2} A_{(n-1,1)}+\omega_{3} A_{(n-3,3)}+\omega_{4} A_{(n-4,3,1)}
$$

for some positive numbers $\omega_{1} \omega_{2}, \omega_{3}$ and $\omega_{4}$ which are to be determined. We are choosing only four of the derangement classes in the association scheme (namely the conjugacy classes with cycle types $(n),(n-1,1),(n-3,3)$ and $(n-4,3,1))$ to have a non-zero weighting. The sizes of the conjugacy classes with cycle types $(n),(n-1,1),(n-3,3)$ and $(n-4,3,1)$ are, respectively,

$$
\alpha=(n-1) !, \quad \beta=n(n-2) !, \quad \gamma=2\left(\begin{array}{l}
n \\
3
\end{array}\right)(n-4) !, \quad \delta=8\left(\begin{array}{l}
n \\
4
\end{array}\right)(n-5) !
$$

We choose our weighting so that the following three conditions hold:

(a) the trivial representation gives the eigenvalue $\left(\begin{array}{l}n \\ 2\end{array}\right)-1$;

(b) the nontrivial irreducible characters that are in the decomposition of the permutation character (namely $\chi^{[n-1,1]}$ and $\chi^{[n-2,2]}$ ) have eigenvalue -1 ; and

(c) all other representations give eigenvalues strictly between -1 and $\left(\begin{array}{l}n \\ 2\end{array}\right)-1$.

It is straightforward to calculate the eigenvalues of the adjacency matrices for the four conjugacy classes we have chosen corresponding to the irreducible characters in the decomposition of the permutation character. These values are in the following table.

\begin{tabular}{|c|c|c|c|c|}
\hline Representation & $A_{(n)}$ & $A_{(n-1,1)}$ & $A_{(n-3,3)}$ & $A_{(n-4,3,1)}$ \\
\hline$\chi^{[n]}$ & $\alpha$ & $\beta$ & $\gamma$ & $\delta$ \\
\hline$\chi^{[n-1,1]}$ & $-\frac{\alpha}{n-1}$ & 0 & $-\frac{\gamma}{n-1}$ & 0 \\
\hline$\chi^{[n-2,2]}$ & 0 & $-\frac{2 \beta}{n(n-3)}$ & 0 & $-\frac{2 \delta}{n(n-3)}$ \\
\hline
\end{tabular}

Table 1: Eigenvalues of $A_{(n)}, A_{(n-1,1)}, A_{(n-3,3)}$ and $A_{(n-4,3,1)}$ afforded by $\chi^{[n]}, \chi^{[n-1,1]}$ and $\chi^{[n-2,2]}$.

By Lemma 10, to find weightings that satisfy conditions (a) and (b) above we need to solve the following three linear equations.

$$
\begin{aligned}
\omega_{1} \alpha+\omega_{2} \beta+\omega_{3} \gamma+\omega_{4} \delta & =\left(\begin{array}{l}
n \\
2
\end{array}\right)-1 \\
-\omega_{1} \alpha-\omega_{3} \gamma & =-(n-1) \\
-\omega_{2} \beta-\omega_{4} \delta & =-\frac{n(n-3)}{2} .
\end{aligned}
$$


This linear system has infinitely many solutions with two free variables. The general solution to (4) has the form

$$
\begin{aligned}
& \omega_{1}(s, t)=\frac{1}{\alpha}(-\gamma s+(n-1)) \\
& \omega_{2}(s, t)=\frac{1}{\beta}\left(-\delta t+\frac{n(n-3)}{2}\right) \\
& \omega_{3}(s, t)=s \\
& \omega_{4}(s, t)=t .
\end{aligned}
$$

Thus, expressed in these two free variables, any eigenvalue of $A$ is of the form

$$
\xi_{\chi}=\frac{1}{\chi(\mathrm{id})}\left(((n-1)-\gamma s) \chi_{(n)}+\left(\frac{n(n-3)}{2}-\delta t\right) \chi_{(n-1,1)}+\gamma s \chi_{(n-3,3)}+\delta t \chi_{(n-4,3,1)}\right)
$$

for $\chi \in \operatorname{Irr}(\operatorname{Sym}(n))$. In other words, eigenvalues of $A$ are functions of the parameters $s$ and $t$. For the remaining part of the proof, we shall write the eigenvalues for an irreducible character $\chi$ in function of the parameters $s$ and $t$ (that is, $\xi_{\chi}(s, t)$ ). We will choose values of $s$ and $t$ so that all the eigenvalues of $A$ satisfy all three conditions (a), (b), and (c).

To do this, we will define a polytope $\mathcal{P}$ and show for any values of $(s, t)$ in $\mathcal{P}$, the weightings $\omega_{i}(s, t)$, for $\mathrm{i} \in\{1,2,3,4\}$, give a matrix $A$ that satisfies the three conditions above. We then apply Theorem 8 to this $A$, which shows that Theorem 4 holds.

Define $\mathcal{P}$ to be the polytope that is the intersection of the following half-spaces of $\mathbb{R}^{2}$

$$
\mathcal{P}\left\{\begin{array}{l}
2 \gamma x-2 \delta y+\left(\begin{array}{c}
n-1 \\
2
\end{array}\right)-(n-1)+2<0 \\
2 \gamma x-2 \delta y+\left(\begin{array}{c}
n-1 \\
2
\end{array}\right)-(n-1)>0 \\
0<\gamma x<n-1, \\
0<\delta y<\frac{n(n-3)}{2} .
\end{array}\right.
$$

The polytope $\mathcal{P}$ is non-empty since the first two equations are those of parallel lines and they intersect the rectangle formed by the last two equations. Note that the final two equations imply the following result.

Lemma 16. For any $(s, t) \in \mathcal{P}$, the weightings $\omega_{1}(s, t), \omega_{2}(s, t), \omega_{3}(s, t)$ and $\omega_{4}(s, t)$ are positive.

Next we will determine the eigenvalues of $A$ so that Theorem 8 can be applied.

Lemma 17. Let $n \geqslant 11$. For any $(s, t) \in \mathcal{P}$, the eigenvalues of the matrix

$$
A=\omega_{1}(s, t) A_{(n)}+\omega_{2}(s, t) A_{(n-1,1)}+\omega_{3}(s, t) A_{(n-3,3)}+\omega_{4}(s, t) A_{(n-4,3,1)}
$$

are in $\left[-1,\left(\begin{array}{l}n \\ 2\end{array}\right)-1\right]$. Moreover, the eigenvalues for $\chi^{[n-1,1]}$ and $\chi^{[n-2,2]}$ are the only ones equal to -1 and the eigenvalue for $\chi^{[n]}$ is the only one equal to $\left(\begin{array}{l}n \\ 2\end{array}\right)-1$. 
Proof. Let $\chi \in \operatorname{Irr}(\operatorname{Sym}(n))$ be such that $\chi(\mathrm{id})>\left(\begin{array}{l}n \\ 2\end{array}\right)$. Using the triangle inequality and Lemma 16 on (5)

$$
\begin{aligned}
\left|\xi_{\chi}\right| & \leqslant \frac{1}{\left(\begin{array}{c}
n \\
2
\end{array}\right)}\left(((n-1)-\gamma s)\left|\chi_{(n)}\right|+\left(\frac{n(n-3)}{2}-\delta t\right)\left|\chi_{(n-1,1)}\right|+\gamma s\left|\chi_{(n-3,3)}\right|+\delta t\left|\chi_{(n-4,3,1)}\right|\right) \\
& \leqslant \frac{1}{\left(\begin{array}{c}
n \\
2
\end{array}\right)}\left(((n-1)-\gamma s)+\left(\frac{n(n-3)}{2}-\delta t\right)+\gamma s+\delta t\right) \\
& =\frac{1}{\left(\begin{array}{c}
n \\
2
\end{array}\right)}\left((n-1)+\frac{n(n-3)}{2}\right) \\
& =\frac{1}{\left(\begin{array}{c}
n \\
2
\end{array}\right)}\left(\left(\begin{array}{l}
n \\
2
\end{array}\right)-1\right) \\
& <1 .
\end{aligned}
$$

Using Lemma 12, the eigenvalues for irreducible characters of dimension less than $\left(\begin{array}{l}n \\ 2\end{array}\right)$ are

$$
\begin{aligned}
\xi_{\chi^{[n]}} & =\left(\begin{array}{l}
n \\
2
\end{array}\right)-1, \\
\xi_{\chi^{[n-1,1]}} & =\xi_{\chi^{[n-2,2]}}=-1 \\
\xi_{\chi^{\left[n-2,1^{2}\right]}} & =\frac{2}{n-2} \\
\xi_{\chi^{\left[3,1^{n-3}\right]}} & =\frac{2(-1)^{n-1}}{n-2}+(-1)^{n} \frac{2 \gamma s}{\left(\begin{array}{c}
n-1 \\
2
\end{array}\right)} \\
\xi_{\chi^{\left[2^{2}, 1^{n-4}\right]}} & =(-1)^{n-1}+(-1)^{n} \frac{4 \delta t}{n(n-3)} \\
\xi_{\chi^{\left[2,1^{n-2}\right]}} & =(-1)^{n}+(-1)^{n-1} \frac{2 \gamma s}{n-1} \\
\xi_{\chi^{\left[1^{n}\right]}} & =(-1)^{n}\left(\left(\begin{array}{l}
n \\
2
\end{array}\right)-2(n-1)-1+2 \gamma s-2 \delta t\right)
\end{aligned}
$$

One can immediately see that, with the exception of $\xi_{\chi^{[n]}}$, these eigenvalues are all strictly less than $\left(\begin{array}{l}n \\ 2\end{array}\right)-1$.

So we need to show that the eigenvalues $\xi_{\chi^{\left[3,1^{n-3}\right]}}, \xi_{\chi^{\left[2^{2}, 1^{n-4}\right]}}, \xi_{\chi^{\left[2,1^{n-2}\right]}}$ and $\xi_{\chi^{\left[1^{n}\right]}}$ are strictly greater than -1 whenever $(s, t) \in \mathcal{P}$.

When $n$ is even, the eigenvalues are larger than -1 if

1. $2 \gamma s-2 \delta t+\left(\begin{array}{c}n-1 \\ 2\end{array}\right)-(n-1)>0$,

2. $\gamma s<n-1$,

3. $0<t$ 
When $n$ is odd, the eigenvalues are larger than -1 if

1. $2 \gamma s-2 \delta t+\left(\begin{array}{c}n-1 \\ 2\end{array}\right)-(n-1)+2<0$,

2. $0<s<\frac{n(n-1)}{4 \gamma}$ (comes from $\left.\left[3,1^{n-3}\right]\right)$,

3. $\delta t<\frac{n(n-3)}{2}$.

Combining the cases when $n$ is even and odd, we obtain exactly the equations of $\mathcal{P}$. Since $\mathcal{P}$ is not empty, all the eigenvalues are greater than -1 for any $(s, t) \in \mathcal{P}$. This completes the proof.

Proof of Theorem 4. For $2 \leqslant n \leqslant 10$, we use Sagemath [20] to prove the result.

For $n \geqslant 11$, we use Theorem 8 , we have

$$
\alpha\left(\Gamma_{\operatorname{Sym}(n)}\right) \leqslant \alpha(X) \leqslant \frac{n !}{1-\frac{\left(\begin{array}{l}
n \\
2
\end{array}\right)-1}{-1}}=2(n-2) ! .
$$

The second part of the proof of Theorem 4 follows immediately from the equality in the ratio bound (see Theorem 8); thus, if $S$ is a maximum 2-setwise intersecting set, then $v_{S}-\left(\left(\begin{array}{l}n \\ 2\end{array}\right)\right)^{-1} \mathbf{1}$ is a -1 -eigenvector for $A$. Since for $n \geqslant 6$, the only irreducible representations that give the least eigenvalue -1 are $\chi^{[n-1,1]}$ and $\chi^{[n-2,2]}$, we have the following corollary.

Corollary 18. For $n \geqslant 6$, any characteristic vector of a maximum 2-setwise intersecting set of permutations in $\operatorname{Sym}(n)$ is in the module $V_{[n]} \oplus V_{[n-1,1]} \oplus V_{[n-2,2]}$.

We note that in $\operatorname{Sym}(4)$ the Klein four group is a 2-setwise intersecting subgroup, and in $\operatorname{Sym}(5)$, the subgroup Alt(4) is a 2-setwise intersecting subgroup. Neither characteristic vectors of these subgroups lie in $V_{[n]} \oplus V_{[n-1,1]} \oplus V_{[n-2,2]}$. So the Corollary does not hold for $n=4,5$.

\section{$5 \quad$ Proof of Theorem 5}

In this section we prove Theorem 5 by constructing a weighted adjacency matrix for the derangement graph of $\operatorname{Sym}(n)$ with the 2-pointwise action. This weighted adjacency matrix will be a linear combination of the adjacency matrices in the conjugacy class scheme for $\operatorname{Sym}(n)$ corresponding to the conjugacy classes with cycle types $(n),(n-1,1)$, $(n-2,2),(n-3,3),(n-3,2,1)$ and $(n-4,3,1)$. In particular, we set

$$
A=\omega_{1} A_{(n)}+\omega_{2} A_{(n-1,1)}+\omega_{3} A_{(n-2,2)}+\omega_{4} A_{(n-3,3)}+\omega_{5} A_{(n-3,2,1)}+\omega_{6} A_{(n-4,3,1)} .
$$

Similar to the proof of Theorem 4 , we will find $\omega_{i}$ for $i=1, \ldots, 6$ so that the following three conditions hold:

(i) the trivial representation gives the eigenvalue $2\left(\begin{array}{l}n \\ 2\end{array}\right)-1$; 
(ii) the nontrivial irreducible characters that are in the decomposition of the 2-pointwise permutation character (namely $\chi^{[n-1,1]}, \chi^{[n-2,2]}$ and $\chi^{[n-2,1,1]}$ ) have eigenvalue -1 ; and

(iii) all other irreducible characters give eigenvalues strictly between $2\left(\begin{array}{l}n \\ 2\end{array}\right)-1$ and -1 .

Define

$$
\begin{array}{rrr}
\alpha=(n-1) !, & \beta=n(n-2) !, & \gamma=\left(\begin{array}{l}
n \\
2
\end{array}\right)(n-3) !, \\
\delta=2\left(\begin{array}{l}
n \\
3
\end{array}\right)(n-4) !, & \mu=3\left(\begin{array}{l}
n \\
3
\end{array}\right)(n-4) !, & \nu=8\left(\begin{array}{l}
n \\
4
\end{array}\right)(n-5) ! .
\end{array}
$$

These numbers are respectively the sizes of the conjugacy classes with cycle type $(n)$, $(n-1,1),(n-2,2),(n-3,3),(n-3,2,1)$ and $(n-4,3,1)$. The following table gives the eigenvalues of the matrices $A_{(n)}, A_{(n-1,1)}, A_{(n-2,2)}, A_{(n-3,3)}, A_{(n-3,2,1)}$ and $A_{(n-4,3,1)}$ in the conjugacy class association scheme corresponding to these irreducible characters.

\begin{tabular}{|c|c|c|c|c|c|c|}
\hline Representation & $A_{(n)}$ & $A_{(n-1,1)}$ & $A_{(n-2,2)}$ & $A_{(n-3,3)}$ & $A_{(n-3,2,1)}$ & $A_{(n-4,3,1)}$ \\
\hline$\chi^{[n]}$ & $\alpha$ & $\beta$ & $\gamma$ & $\delta$ & $\mu$ & $\nu$ \\
\hline$\chi^{[n-1,1]}$ & $-\frac{\alpha}{n-1}$ & 0 & $-\frac{\gamma}{n-1}$ & $\frac{-\delta}{n-1}$ & 0 & 0 \\
\hline$\chi^{[n-2,2]}$ & 0 & $-\frac{2 \beta}{n(n-3)}$ & $\frac{2 \gamma}{n(n-3)}$ & 0 & 0 & $-\frac{2 \nu}{n(n-3)}$ \\
\hline$\chi^{[n-2,1,1]}$ & $\frac{\alpha}{\left(\begin{array}{c}n-1 \\
2\end{array}\right)}$ & 0 & 0 & $\frac{\delta}{\left(\begin{array}{c}n-1 \\
2\end{array}\right)}$ & $-\frac{\mu}{\left(\begin{array}{c}n-1 \\
2\end{array}\right)}$ & 0 \\
\hline
\end{tabular}

Table 2: Eigenvalues afforded by $\chi^{[n]}, \chi^{[n-1,1]}, \chi^{[n-2,2]}$ and $\chi^{[n-2,1,1]}$ on $A_{(n)}, A_{(n-1,1)}$, $A_{(n-2,2)}, A_{(n-3,3)}, A_{(n-3,2,1)}$ and $A_{(n-4,3,1)}$.

Using Lemma 10, it is straightforward to calculate the eigenvalues of $A$ afforded by $\chi^{[n]}, \chi^{[n-1,1]}, \chi^{[n-2,2]}$ and $\chi^{[n-2,1,1]}$ as functions of $\omega_{i}$ where $i=1, \ldots, 6$. Thus, in order to satisfy conditions (i) and (ii) above, the $\omega_{i}$ must satisfy the following system of linear equations.

$$
\begin{aligned}
\alpha \omega_{1}+\beta \omega_{2}+\gamma \omega_{3}+\delta \omega_{4}+\mu \omega_{5}+\nu \omega_{6} & =n(n-1)-1 \\
-\alpha \omega_{1}-\gamma \omega_{3}-\delta \omega_{4} & =-(n-1) \\
-\beta \omega_{2}+\gamma \omega_{3}-\nu \omega_{6} & =-\frac{n(n-3)}{2} \\
\alpha \omega_{1}+\delta \omega_{4}-\mu \omega_{5} & =-\left(\begin{array}{c}
n-1 \\
2
\end{array}\right) .
\end{aligned}
$$

The system (7) has infinitely many solutions with three free variables. The following is a general solution to (7). 


$$
\begin{aligned}
& \omega_{1}(r, s, t)=\frac{1}{\alpha}\left(\left(\begin{array}{l}
n \\
2
\end{array}\right)-1-\beta r-\nu t-\delta s\right), \\
& \omega_{2}(r, s, t)=r, \\
& \omega_{3}(r, s, t)=\frac{1}{\gamma}\left(1-\left(\begin{array}{c}
n-1 \\
2
\end{array}\right)+\beta r+\nu t\right), \\
& \omega_{4}(r, s, t)=s, \\
& \omega_{5}(r, s, t)=\frac{1}{\mu}\left(\left(\begin{array}{l}
n \\
2
\end{array}\right)+\left(\begin{array}{c}
n-1 \\
2
\end{array}\right)-1-\beta r-\nu t\right) \\
& \omega_{6}(r, s, t)=t
\end{aligned}
$$

for $r, s, t \in \mathbb{R}$.

The eigenvalue of $A$ corresponding to $\chi$, denoted by $\xi_{\chi}(r, s, t)$, as a function of $r, s$, and $t$ is the following.

$$
\begin{aligned}
\xi_{\chi}(r, s, t)= & \frac{1}{\chi(\mathrm{id})}\left(\left(\left(\begin{array}{l}
n \\
2
\end{array}\right)-1-\beta r-\delta s-\nu t\right) \chi_{(n)}+\beta r \chi_{(n-1,1)}\right. \\
& +\left(1-\left(\begin{array}{c}
n-1 \\
2
\end{array}\right)+\beta r+\nu t\right) \chi_{(n-2,2)}+\delta s \chi_{(n-3,3)} \\
& \left.+\left(\left(\begin{array}{l}
n \\
2
\end{array}\right)+\left(\begin{array}{c}
n-1 \\
2
\end{array}\right)-1-\beta r-\nu t\right) \chi_{(n-3,2,1)}+\nu t \chi_{(n-4,3,1)}\right) .
\end{aligned}
$$

In particular, we have

$$
\begin{aligned}
\xi_{\chi^{\left[1^{n}\right]}} & =(-1)^{n}\left(4 \beta r+2 \nu t+2 \delta s+3-2\left(\begin{array}{l}
n \\
2
\end{array}\right)-2\left(\begin{array}{c}
n-1 \\
2
\end{array}\right)\right) \\
\xi_{\chi^{\left[2,1^{n-2}\right]}} & =\frac{(-1)^{n}}{n-1}\left(\left(\begin{array}{l}
n \\
2
\end{array}\right)+\left(\begin{array}{c}
n-1 \\
2
\end{array}\right)-2-2 \beta r-2 \delta s-2 \nu t\right) \\
\xi_{\chi^{\left[2^{2}, 1^{n-4}\right]}} & =(-1)^{n-1}+(-1)^{n} \frac{4 \nu t}{n(n-3)} \\
\xi_{\left.\chi^{[3,1} 1^{n-3}\right]} & =(-1)^{n}+(-1)^{n} \frac{2 \delta s}{\left(\begin{array}{c}
n-1 \\
2
\end{array}\right)} .
\end{aligned}
$$

We will distinguish the cases $n$ even and $n$ odd. For each case we will pick values for $r, s$ and $t$ so that the matrix $A$ satisfies the conditions in Theorem 8 .

\subsection{Subcase 1: $n$ even}

For this case we set $s=0$; this removes the conjugacy class with cycle type $(n-3,3)$ from the weighted adjacency matrix. With $s=0$ and $n$ even, we can calculate the eigenvalues 
for the eight irreducible characters of dimension less than $2\left(\begin{array}{c}n+1 \\ 2\end{array}\right)$ as follows.

$$
\begin{aligned}
\xi_{\chi^{[n]}} & =2\left(\begin{array}{l}
n \\
2
\end{array}\right)-1 \\
\xi_{\chi^{[n-1,1]}} & =\xi_{\chi^{[n-2,2]}}=\xi_{\chi^{\left[n-2,1^{2}\right]}}=-1 \\
\xi_{\chi^{\left[3,1^{n-3}\right]}} & =1 \\
\xi_{\chi^{\left[2^{2}, 1^{n-4}\right]}} & =-1+\frac{4 \nu t}{n(n-3)} \\
\xi_{\chi^{\left[2,1^{n-2}\right]}} & =\frac{1}{n-1}\left(\left(\begin{array}{c}
n \\
2
\end{array}\right)+\left(\begin{array}{c}
n-1 \\
2
\end{array}\right)-2-2 \beta r-2 \nu t\right) \\
\xi_{\chi^{\left[1^{n}\right]}} & =4 \beta r+2 \nu t+3-2\left(\begin{array}{c}
n \\
2
\end{array}\right)-2\left(\begin{array}{c}
n-1 \\
2
\end{array}\right)
\end{aligned}
$$

Let $\mathcal{P}^{\prime}$ be the polytope obtained by the following equations of half-spaces of $\mathbb{R}^{2}$

$$
\mathcal{P}^{\prime}\left\{\begin{array}{l}
2 \beta x+\nu y-\left(\begin{array}{l}
n \\
2
\end{array}\right)-\left(\begin{array}{c}
n-1 \\
2
\end{array}\right)+2>0 \\
\beta x+\nu y+1-\left(\begin{array}{l}
n \\
2
\end{array}\right)<0 \\
0<y .
\end{array}\right.
$$

This polytope is defined so that the eigenvalues $\xi_{\chi^{\left[2^{2}, 1^{n-4}\right]}}(r, 0, t), \xi_{\chi^{\left[2,1^{n-2}\right]}}(r, 0, t)$ and $\xi_{\chi^{\left[1^{n}\right]}}(r, 0, t),(r, t) \in \mathcal{P}^{\prime}$, are all strictly greater than -1 . We let the reader verify that $\mathcal{P}^{\prime}$ is a triangle (without the boundary) with coordinates

$$
\left(\frac{1}{\beta}\left(\left(\begin{array}{c}
n-1 \\
2
\end{array}\right)-1\right), \frac{1}{\nu}(n-2)\right), \quad\left(\frac{1}{\beta}\left(\left(\begin{array}{l}
n \\
2
\end{array}\right)-1\right), 0\right), \quad\left(\frac{1}{\beta}\left(\left(\begin{array}{c}
n-1 \\
2
\end{array}\right)-1+\frac{n-1}{2}\right), 0\right) .
$$

In particular, $\mathcal{P}^{\prime}$ is non-empty.

Next, we prove that for any $(r, t) \in \mathcal{P}^{\prime}$, the weightings $w_{i}$ are all non-negative.

Lemma 19. The weighting $\omega_{i}(r, 0, t)$ is non-negative for any $i=1, \ldots, 6$ and $(r, t) \in \mathcal{P}^{\prime}$.

Proof. Let $(r, t) \in \mathcal{P}^{\prime}$. From the equations of $\mathcal{P}^{\prime}$, we have

$$
-\beta x-\nu y>1-\left(\begin{array}{l}
n \\
2
\end{array}\right), \quad \beta x+\nu y>2\left(\begin{array}{l}
n \\
2
\end{array}\right)+2\left(\begin{array}{c}
n-1 \\
2
\end{array}\right)-2-\beta x .
$$

Using these relations and the fact that $\beta x \in\left[\left(\begin{array}{c}n-1 \\ 2\end{array}\right)-1,\left(\begin{array}{c}n \\ 2\end{array}\right)-1\right]$, one can derive that the weightings are indeed positive.

Next, we prove that the eigenvalues of the weighted adjacency matrix are also in the correct range whenever $(r, t) \in \mathcal{P}^{\prime}$. 
Lemma 20. Let $n \geqslant 13$ and even. If $(r, t) \in \mathcal{P}^{\prime}$, then the eigenvalues of the weighted adjacency matrix $A$ defined in (6) are in the interval $\left[-1,2\left(\begin{array}{c}n \\ 2\end{array}\right)-1\right]$. In addition, the only irreducible character giving the eigenvalue $2\left(\begin{array}{l}n \\ 2\end{array}\right)-1$ is $\chi^{[n]}$; and the only irreducible characters giving eigenvalue -1 are $\chi^{[n-1,1]}, \chi^{[n-2,2]}$ and $\chi^{\left[n-2,1^{2}\right]}$.

Proof. First we show that the statement holds for all irreducible characters $\chi$ of $\operatorname{Sym}(n)$ with $\chi($ id $)>2\left(\begin{array}{c}n+1 \\ 2\end{array}\right)$. On each of the conjugacy classes that we consider, the value of any irreducible character is bounded by 1 . For any $(r, t) \in \mathcal{P}^{\prime}$ we have the following bound.

$$
\begin{aligned}
& \left|\xi_{\chi}(r, 0, t)\right| \leqslant \frac{1}{2\left(\begin{array}{c}
n+1 \\
2
\end{array}\right)}\left(\left(\begin{array}{l}
n \\
2
\end{array}\right)-1-\beta r-\nu t\right)\left|\chi_{(n)}\right|+\beta r\left|\chi_{(n-1,1)}\right| \\
& +\left(1-\left(\begin{array}{c}
n-1 \\
2
\end{array}\right)+\beta r+\nu t\right)\left|\chi_{(n-2,2)}\right| \\
& \left.+\left(\left(\begin{array}{l}
n \\
2
\end{array}\right)+\left(\begin{array}{c}
n-1 \\
2
\end{array}\right)-1-\beta r-\nu t\right)\left|\chi_{(n-3,2,1)}\right|+\nu t\left|\chi_{(n-4,3,1)}\right|\right) \\
& =\frac{2\left(\begin{array}{c}
n \\
2
\end{array}\right)-1}{2\left(\begin{array}{c}
n+1 \\
2
\end{array}\right)}<1 \text {. }
\end{aligned}
$$

Thus, the eigenvalue of $A$ for $\chi$ is bounded in absolute value by 1 and the statement holds for all irreducible characters except for those corresponding to the partitions $[n],\left[1^{n}\right]$, $[n-1,1],\left[2,1^{n-2}\right],[n-2,2],\left[2^{2}, 1^{n-4}\right],\left[n-2,1^{2}\right]$ or $\left[3,1^{n-3}\right]$.

It is straightforward to see that the non-trivial characters all give eigenvalue less than $2\left(\begin{array}{l}n \\ 2\end{array}\right)-1$.

Finally, (11) and the definition of $\mathcal{P}^{\prime}$ show that the statement holds for all other irreducible characters.

\section{$5.2 \quad$ Subcase 2: $n$ odd}

If $n$ is odd, we first note that in the expression of the eigenvalue for $\left[3,1^{n-3}\right]$ in $(10)$, the value of $s$ must be negative for the inequality $\xi_{\chi^{\left[3,1^{n-3}\right]}}>-1$ to hold. We will only use five conjugacy classes, so we drop conjugacy classes with cycle type $(n-4,3,1)$ by making $t=0$. As in the previous case, we will consider the irreducible characters with dimension less than $2\left(\begin{array}{c}n+1 \\ 2\end{array}\right)$ and greater than $2\left(\begin{array}{c}n+1 \\ 2\end{array}\right)$ separately. The eigenvalues belonging to representations with dimension less than $2\left(\begin{array}{c}n+1 \\ 2\end{array}\right)$ are

$$
\begin{aligned}
\xi_{\chi^{[n]}} & =2\left(\begin{array}{c}
n \\
2
\end{array}\right)-1 . \\
\xi_{\chi^{[n-1,1]}} & =\xi_{\chi^{[n-2,2]}}=\xi_{\chi^{\left[n-2,1^{2}\right]}}=-1 \\
\xi_{\chi^{\left[3,1^{n-3}\right]}} & =-1-\frac{2 \delta s}{\left(\begin{array}{c}
n-1 \\
2
\end{array}\right)} \\
\xi_{\chi^{\left[2^{2}, 1^{n-4}\right]}} & =1 \\
\xi_{\chi^{\left[2,1^{n-2}\right]}} & =\frac{-1}{n-1}\left(\left(\begin{array}{c}
n \\
2
\end{array}\right)+\left(\begin{array}{c}
n-1 \\
2
\end{array}\right)-2-2 \beta r-2 \delta s\right)
\end{aligned}
$$




$$
\xi_{\chi^{\left[1^{n}\right]}}=-\left(4 \beta r+2 \delta s+3-2\left(\begin{array}{l}
n \\
2
\end{array}\right)-2\left(\begin{array}{c}
n-1 \\
2
\end{array}\right)\right)
$$

Let $\mathcal{P}^{\prime \prime}$ be the polytope of $\mathbb{R}^{2}$ defined as follows.

$$
\mathcal{P}^{\prime \prime}\left\{\begin{array}{l}
2 \beta x+\delta y+1-\left(\begin{array}{c}
n \\
2
\end{array}\right)-\left(\begin{array}{c}
n-1 \\
2
\end{array}\right)<0 \\
\beta x+\delta y+1-\left(\begin{array}{c}
n-1 \\
2
\end{array}\right)>0 \\
y<0 .
\end{array}\right.
$$

Just as in the case when $n$ is even, this polytope is defined so that the eigenvalues $\xi_{\chi^{\left[2^{2}, 1^{n-4}\right]}}$, $\xi_{\chi^{\left[2,1^{n-2}\right]}}$ and $\xi_{\chi^{\left[1^{n}\right]}}$ are all strictly greater than -1 . The polytope $\mathcal{P}^{\prime \prime}$ is a triangle (without the boundary) with coordinates

$$
\left(\frac{1}{\beta}\left(\begin{array}{l}
n \\
2
\end{array}\right), \frac{-n}{\delta}\right), \quad\left(\frac{1}{\beta}\left(\left(\begin{array}{c}
n-1 \\
2
\end{array}\right)-1\right), 0\right), \quad\left(\frac{1}{2 \beta}\left(\left(\begin{array}{l}
n \\
2
\end{array}\right)+\left(\begin{array}{c}
n-1 \\
2
\end{array}\right)-1\right), 0\right) .
$$

In the next lemma, we state that all weightings, except $\omega_{4}$, are positive for any $(r, s) \in$ $\mathcal{P}^{\prime \prime}$. We give the statement of this without a proof since it is straightforward.

Lemma 21. For $i \in\{1,2,3,5,6\}$ and for $(r, s) \in \mathcal{P}^{\prime \prime}$, we have $\omega_{i}(r, s, 0) \geqslant 0$.

As in the even case, the eigenvalues of the weighted adjacency matrix are in the required range whenever $(r, s) \in \mathcal{P}^{\prime \prime}$.

Lemma 22. Let $n \geqslant 13$ and odd. For $(r, s) \in \mathcal{P}^{\prime \prime}$, the eigenvalues of the weighted adjacency matrix $A$ defined in (6) are in $\left[-1,2\left(\begin{array}{l}n \\ 2\end{array}\right)-1\right]$. Moreover, the eigenvalue $2\left(\begin{array}{l}n \\ 2\end{array}\right)-$ 1 is afforded only by $\chi^{[n]}$ and the only irreducible characters giving eigenvalue -1 are $\chi^{[n-1,1]}, \chi^{\left[n-2,1^{2}\right]}$ and $\chi^{[n-2,2]}$.

Proof. Lemma 12 gives the eight irreducible representations of $\operatorname{Sym}(n)$ with dimension less than $2\left(\begin{array}{c}n+1 \\ 2\end{array}\right)$. The polytope $\mathcal{P}^{\prime \prime}$ is defined so that this result holds for these eight irreducible characters.

Next assume $\chi$ is an irreducible character of $\operatorname{Sym}(n)$ with $\chi(\mathrm{id})>2\left(\begin{array}{c}n+1 \\ 2\end{array}\right)$. On each of the conjugacy classes that we consider, the value of any irreducible representation is bounded by 1 . Noting that $(r, s) \in \mathcal{P}^{\prime \prime}$ implies $\delta s \in(-n, 0)$, we have the following bound.

$$
\begin{aligned}
\left|\xi_{\chi}(r, s, 0)\right| \leqslant & \frac{1}{\chi(\mathrm{id})}\left(\left(\begin{array}{l}
n \\
2
\end{array}\right)-1-\beta r-\delta s\right)\left|\chi_{(n)}\right| \\
& +\beta r\left|\chi_{(n-1,1)}\right|+\left(1-\left(\begin{array}{c}
n-1 \\
2
\end{array}\right)+\beta r\right)\left|\chi_{(n-2,2)}\right| \\
& +|\delta s|\left|\chi_{(n-3,3)}\right|+\left(\left(\begin{array}{c}
n \\
2
\end{array}\right)+\left(\begin{array}{c}
n-1 \\
2
\end{array}\right)-1-\beta r\right)\left|\chi_{(n-3,2,1)}\right| \\
\leqslant & \frac{1}{2\left(\begin{array}{c}
n+1 \\
2
\end{array}\right)}\left(\left(\begin{array}{l}
n \\
2
\end{array}\right)-1-\beta r+n\right)+\beta r+\left(1-\left(\begin{array}{c}
n-1 \\
2
\end{array}\right)+\beta r\right)
\end{aligned}
$$




$$
\begin{aligned}
& +n+\left(\left(\begin{array}{l}
n \\
2
\end{array}\right)+\left(\begin{array}{c}
n-1 \\
2
\end{array}\right)-1-\beta r\right) . \\
\leqslant & \frac{1}{2\left(\begin{array}{c}
n+1 \\
2
\end{array}\right)}\left(2\left(\begin{array}{l}
n \\
2
\end{array}\right)-1+2 n\right) \\
< & \frac{n^{2}+n-1}{n^{2}+n} \\
< & 1
\end{aligned}
$$

Thus, the eigenvalue corresponding to any irreducible character with dimension greater than or equal to $2\left(\begin{array}{c}n+1 \\ 2\end{array}\right)$ is strictly between -1 and 1 , so the result holds.

The proof of Theorem 5 follows from Lemma 20 and Lemma 22 using the ratio bound on $\Gamma_{n}$ with the weighted adjacency matrix $A$.

Proof of Theorem 5. If $5 \leqslant n \leqslant 12$, we use Sagemath [20] to confirm that the result holds. For $n \geqslant 13$, we prove the result by using Theorem 8 . Therefore, if $n$ is even, then $\alpha\left(\Gamma_{\operatorname{Sym}(n)}\right) \leqslant \alpha\left(X_{1}\right)=(n-2)$ !, and similarly, if $n$ is odd, then $\alpha\left(\Gamma_{\operatorname{Sym}(n)}\right) \leqslant \alpha\left(X_{2}\right)=$ $(n-2) !$

We finish this section by proving a conjecture of Godsil and Meagher [13, Conjecture 7.3].

Corollary 23. Let $n \geqslant 5$. If $v$ is the characteristic vector of a maximum 2-pointwise intersecting family of $\operatorname{Sym}(n)$, then $v \in V_{[n]} \oplus V_{[n-1,1]} \oplus V_{[n-2,2]} \oplus V_{\left[n-2,1^{2}\right]}$.

\section{Further work}

In this paper we construct weighted adjacency matrices for the derangement graphs of $\operatorname{Sym}(n)$ for two different actions (2-pointwise and 2-setwise). This work proves that the conjectured lower bounds on $n$ for each action are indeed the correct bound when $t=2$. It is interesting that this work also shows that there are infinitely many weighted adjacency matrices that would work in the ratio bound. We leave the reader with two open problems.

Problem 24. In Theorem 4 we proved that any characteristic vector of a maximum 2setwise intersecting family is in the $V_{[n]} \oplus V_{[n-1,1]} \oplus V_{[n-2,2]}$ module. Similarly, Theorem 5, shows that the characteristic vector of any maximum 2-pointwise intersecting family lies in the $V_{[n]} \oplus V_{[n-1,1]} \oplus V_{[n-2,2]} \oplus V_{[n-2,1,1]}$ module. This gives information about the maximum intersecting families for both type of intersections, but we would like to know the exact characterization of the maximum intersecting sets.

In Sym(4), the Klein four group is a 2-setwise intersecting subgroup that is not the stabilizer of a 2-set. Similarly, in Sym(5), the subgroup Alt(4) is a 2-setwise intersecting subgroup that is not the stabilizer of a 2-set, as are all of its cosets. Other than these examples, we conjecture that the maximum 2-setwise intersecting subset of $\operatorname{Sym}(n)$ is either the setwise stabilizer of a set of size 2, or a coset of a setwise stabilizer of a set of size 2 . 
Problem 25. Prove that the $t$-setwise intersecting property of $\operatorname{Sym}(n)$ holds for any $3 \leqslant t \leqslant n$ and for $n \geqslant 2 t+1$, prove that $\operatorname{Sym}(n)$ has the $t$-pointwise intersecting property.

\section{Acknowledgements}

We would like to thank the two anonymous referees for their valuable feedback which greatly helped improve the content of this paper.

\section{References}

[1] László Babai. Spectra of Cayley graphs. Journal of Combinatorial Theory, Series B, 27(2):180-189, 1979.

[2] Peter J. Cameron and Cheng Yeaw Ku. Intersecting families of permutations. European Journal of Combinatorics, 24(7):881-890, 2003.

[3] P. Delsarte. An Algebraic Approach to the Association Schemes of Coding Theory. Philips Res. Rep., Suppl., vol. 10, 1973.

[4] M. Deza and P. Frankl. Erdős-Ko-Rado theorem-22 years later. SIAM Journal on Algebraic Discrete Methods, 4(4):419-431, 1983.

[5] Michel Deza, Paul Erdős, and Péter Frankl. Intersection properties of systems of finite sets. Proceedings of the London Mathematical Society, 3(2):369-384, 1978.

[6] Persi Diaconis and Mehrdad Shahshahani. Generating a random permutation with random transpositions. Z. Wahrsch. Verw. Gebiete, 57(2):159-179, 1981.

[7] David Ellis. Setwise intersecting families of permutations. Journal of Combinatorial Theory, Series A, 119(4):825-849, 2012.

[8] David Ellis, Ehud Friedgut, and Haran Pilpel. Intersecting families of permutations. Journal of the American Mathematical Society, 24(3):649-682, 2011.

[9] P. Erdős, Chao Ko, and R. Rado. Intersection theorems for systems of finite sets. The Quarterly Journal of Mathematics, 12(1):313-320, 1961.

[10] J S. Frame, G. de B. Robinson, and R. M. Thrall. The hook graphs of the symmetric group. Canadian Journal of Mathematics, 6:316-324, 1954.

[11] Peter Frankl and Mikhail Deza. On the maximum number of permutations with given maximal or minimal distance. Journal of Combinatorial Theory, Series A, 22(3):352-360, 1977.

[12] Zoltán Füredi, Kyung-Won Hwang, and Paul M. Weichsel. A proof and generalizations of the Erdös-Ko-Rado theorem using the method of linearly independent polynomials. In Topics in discrete mathematics, pages 215-224. Springer, 2006.

[13] Chris Godsil and Karen Meagher. A new proof of the Erdős-Ko-Rado theorem for intersecting families of permutations. European Journal of Combinatorics, 30(2):404$414,2009$. 
[14] Chris Godsil and Karen Meagher. An algebraic proof of the Erdös-Ko-Rado theorem for intersecting families of perfect matchings. Ars Math. Contemp. 12(2):205-217, 2017.

[15] Chris Godsil and Karen Meagher. Erdös-Ko-Rado Theorems: Algebraic Approaches. Number 149. Cambridge University Press, 2016.

[16] Gyula O. H. Katona. A simple proof of the Erdős-Ko-Rado theorem. Journal of Combinatorial Theory, Series B, 13(2):183-184, 1972.

[17] Benoit Larose and Claudia Malvenuto. Stable sets of maximal size in Kneser-type graphs. European Journal of Combinatorics, 25(5):657-673, 2004.

[18] Karen Meagher and Peter Sin. All 2-transitive groups have the EKR-module property. J. Combin. Theory Ser. A 177 (2021), 21 pp.

[19] B. E. Sagan. The Symmetric Group: Representations, Combinatorial Algorithms, and Symmetric Functions (Graduate Texts in Mathematics). New York: Springer, 2001.

[20] The Sage Developers. SageMath, the Sage Mathematics Software System (Version 8.9), 2020. https://www. sagemath.org. 


\section{A Character values}

\begin{tabular}{|c|c|c|c|c|c|c|c|}
\hline Representation & Range of $k$ & $C_{(n-3,3)}$ & $C_{(n-4,3,1)}$ & $C_{(n)}$ & $C_{(n-1,1)}$ & $C_{(n-2,2)}$ & $C_{(n-3,2,1)}$ \\
\hline$\chi^{\lfloor n\rfloor}$ & - & 1 & 1 & 1 & 1 & 1 & 1 \\
\hline$\chi^{[n-1,1]}$ & - & -1 & 0 & -1 & 0 & -1 & 0 \\
\hline$\chi^{\lfloor n-2,2]}$ & - & 0 & -1 & 0 & -1 & 1 & 0 \\
\hline$\chi^{\left[n-2,1^{2}\right]}$ & - & 1 & 0 & 1 & 0 & 0 & -1 \\
\hline$\chi^{[n-3,3]}$ & - & 1 & 1 & 0 & 0 & -1 & 0 \\
\hline$\chi^{[n-3,2,1]}$ & - & -1 & 0 & 0 & 0 & 0 & 1 \\
\hline$\chi^{\left[n-3,1^{3}\right]}$ & - & 0 & -1 & -1 & 0 & 0 & 0 \\
\hline$\chi^{\left[n-4,2^{2}\right]}$ & - & 1 & 0 & 0 & 0 & -1 & -1 \\
\hline$\chi^{\left[n-4,2,1^{2}\right]}$ & - & 0 & 1 & 0 & -1 & 0 & 0 \\
\hline$\chi^{\left[n-5,2^{2}, 1\right]}$ & - & 0 & -1 & 0 & 0 & 1 & 0 \\
\hline$\chi^{\left[n-6,2^{3}\right]}$ & - & -1 & -1 & 0 & 0 & 0 & 1 \\
\hline$\chi^{\left[n-k-4,4,1^{k}\right]}$ & $0 \leqslant k<n-8$ & $(-1)^{k+1}$ & 0 & 0 & 0 & 0 & $(-1)^{k+1}$ \\
\hline$\chi^{\left[n-k-5,5,1^{k}\right]}$ & $0 \leqslant k \leqslant n-10$ & 0 & $(-1)^{k+1}$ & 0 & 0 & 0 & 0 \\
\hline$\chi^{\left[n-k-5,3,2,1^{k}\right]}$ & $0 \leqslant k \leqslant n-8$ & $(-1)^{k+3}$ & 0 & 0 & 0 & 0 & 0 \\
\hline$\chi^{\left[n-k-6,2^{3}, 1^{k}\right]}$ & $0<k \leqslant n-8$ & $(-1)^{k+3}$ & 0 & 0 & 0 & 0 & $(-1)^{k+4}$ \\
\hline$\chi^{\left[n-k-6,3^{2}, 1^{k}\right]}$ & $0 \leqslant k \leqslant n-9$ & 0 & $(-1)^{k+1}$ & 0 & 0 & 0 & 0 \\
\hline$\chi^{\left[n-k-8,2^{4}, 1^{k}\right]}$ & $0 \leqslant k \leqslant n-10$ & 0 & $(-1)^{k+4}$ & 0 & 0 & 0 & 0 \\
\hline$\chi^{\left[n-k, 1^{k}\right]}$ & $4 \leqslant k \leqslant n-5$ & 0 & 0 & $(-1)^{k}$ & 0 & 0 & 0 \\
\hline$\chi^{\left[n-k-2,2,1^{k}\right]}$ & $3 \leqslant k \leqslant n-6$ & 0 & 0 & 0 & $(-1)^{k+1}$ & 0 & 0 \\
\hline$\chi^{\left[4^{2}, 1^{n-8}\right]}$ & - & $(-1)^{n-7}$ & $(-1)^{n-6}$ & 0 & 0 & 0 & $(-1)^{n-7}$ \\
\hline$\chi^{\left[4,3,1^{n-7}\right]}$ & - & 0 & $(-1)^{n-5}$ & 0 & 0 & $(-1)^{n-6}$ & 0 \\
\hline$\chi^{\left[4,2,1^{n-6}\right]}$ & - & 0 & $(-1)^{n-4}$ & 0 & $(-1)^{n-5}$ & 0 & 0 \\
\hline$\chi^{\left[3^{2}, 1^{n-6}\right]}$ & - & $(-1)^{n-4}$ & 0 & 0 & 0 & $(-1)^{n-5}$ & $(-1)^{n-6}$ \\
\hline$\chi^{\left[4,1^{n-4}\right]}$ & - & 0 & $(-1)^{n-4}$ & $(-1)^{n-4}$ & 0 & 0 & 0 \\
\hline$\chi^{\left[3,2,1^{n-5}\right]}$ & - & $(-1)^{n-3}$ & 0 & 0 & $(-1)^{n-4}$ & 0 & $(-1)^{n-5}$ \\
\hline$\chi^{\left[2^{3}, 1^{n-6}\right]}$ & - & $(-1)^{n-2}$ & $(-1)^{n-3}$ & 0 & $(-1)^{n-5}$ & $(-1)^{n-3}$ & 0 \\
\hline$\chi^{\left[3,1^{n-3}\right]}$ & - & $(-1)^{n-2}$ & 0 & $(-1)^{n-3}$ & 0 & 0 & $(-1)^{n-4}$ \\
\hline$\chi^{\left[2^{2}, 1^{n-4}\right]}$ & - & 0 & $(-1)^{n-4}$ & 0 & $(-1)^{n-3}$ & $(-1)^{n-4}$ & 0 \\
\hline$\chi^{\left[2,1^{n-2}\right]}$ & - & $(-1)^{n-1}$ & 0 & $(-1)^{n-2}$ & 0 & $(-1)^{n-3}$ & 0 \\
\hline$\chi^{\left[1^{n}\right\rfloor}$ & - & $(-1)^{n-2}$ & $(-1)^{n-1}$ & $(-1)^{n-1}$ & $(-1)^{n-2}$ & $(-1)^{n-2}$ & $(-1)^{n-3}$ \\
\hline
\end{tabular}

Table 3: Values of irreducible characters on conjugacy classes $C_{(n-3,3)}, C_{(n-4,3,1)}, C_{(n)}$, $C_{(n-1,1)}, C_{(n-2,2)}$ and $C_{(n-3,2,1)}$. 